\title{
A REMARK ON THE KERNEL OF THE CR PANEITZ OPERATOR
}

\author{
JEFFREY S. CASE, SAGUN CHANILLO, AND PAUL YANG
}

\begin{abstract}
For CR structures in dimension three, the CR pluriharmonic functions are characterized by the vanishing of a third order operator. This third order operator, after composition with the divergence operator, gives the fourth order analogue of the Paneitz operator. In this short note, we give criteria under which the kernel of the CR Paneitz operator contains a supplementary space to the CR pluriharmonic functions.
\end{abstract}

\section{INTRODUCTION}

It is well-known that for a three-dimensional CR manifold, the kernel of the CR Paneitz operator contains the CR pluriharmonic functions. It is an open question whether there are examples of CR manifolds for which the kernel of the CR Paneitz operator is exactly the CR pluriharmonic functions. Graham and Lee showed [6] that this is true for compact CR manifolds admitting a torsion free contact form. More recently, the authors showed [3] that this is true for real analytic families of CR manifolds for which the space of CR pluriharmonic functions is stable, assuming positivity of certain natural geometric invariants. In this short note, we give conditions, related to the instability of the space of CR pluriharmonic functions along deformations, which guarantee that the kernel of the CR Paneitz operator contains functions which are not CR pluriharmonic; see Theorem 2.1 and Theorem 2.3.

Throughout this paper, we use the notation and terminology in [8] unless otherwise specified. Let $(M, J, \theta)$ be a closed three-dimensional pseudohermitian manifold, where $\theta$ is a contact form and $J$ is a CR structure compatible with the contact bundle $\xi=\operatorname{ker} \theta$. The $\mathrm{CR}$ structure $J$ decomposes $\xi \otimes \mathbb{C}$ into the $+i$ - and $-i$ eigenspaces of $J$, denoted $T_{1,0}$ and $T_{0,1}$, respectively. The Levi form $\langle,\rangle_{L_{\theta}}$ is the Hermitian form on $T_{1,0}$ defined by $\langle Z, W\rangle_{L_{\theta}}=-i\langle d \theta, Z \wedge \bar{W}\rangle$. We can extend $\langle,\rangle_{L_{\theta}}$ to $T_{0,1}$ by defining $\langle\bar{Z}, \bar{W}\rangle_{L_{\theta}}=\overline{\langle Z, W}_{L_{\theta}}$ for all $Z, W \in T_{1,0}$. The Levi form induces naturally a Hermitian form on the dual bundle of $T_{1,0}$, denoted by $\langle,\rangle_{L_{\theta}^{*}}$, and hence on all the induced tensor bundles. By integrating the Hermitian form (when acting on sections) over $M$ with respect to the volume form $d V=\theta \wedge d \theta$, we get an inner product on the space of sections of each tensor bundle. We denote this inner product by $\langle$,$\rangle . For example$

$$
\langle\varphi, \psi\rangle=\int_{M} \varphi \bar{\psi} d V
$$

for functions $\varphi$ and $\psi$.

SC was partially supported by NSF Grant No. DMS-1201474.

PY was partially supported by NSF Grant No. DMS-1104536. 
The Reeb vector field $T$ is the unique vector field such that $\theta(T)=1$ and $\theta(T, \cdot)=0$. Let $Z_{1}$ be a local frame of $T_{1,0}$ and consider the frame $\left\{T, Z_{1}, Z_{\overline{1}}\right\}$ of $T M \otimes \mathbb{C}$. Then $\left\{\theta, \theta^{1}, \theta^{\overline{1}}\right\}$, the coframe dual to $\left\{T, Z_{1}, Z_{\overline{1}}\right\}$, satisfies

$$
d \theta=i h_{1 \overline{1}} \theta^{1} \wedge \theta^{\overline{1}}
$$

for some positive function $h_{1 \overline{1}}$. We can always choose $Z_{1}$ such that $h_{1 \overline{1}}=1$; hence, throughout this paper, we assume $h_{1 \overline{1}}=1$

The pseudohermitian connection of $(J, \theta)$ is $\nabla$ on $T M \otimes \mathbb{C}$ (and extended to tensors) given in terms of a local frame $Z_{1} \in T_{1,0}$ by

$$
\nabla Z_{1}=\theta_{1}^{1} \otimes Z_{1}, \quad \nabla Z_{\overline{1}}=\theta_{\overline{1}}^{\overline{1}} \otimes Z_{\overline{1}}, \quad \nabla T=0
$$

where $\theta_{1}{ }^{1}$ is the 1 -form uniquely determined by the equations

$$
\begin{aligned}
d \theta^{1} & =\theta^{1} \wedge \theta_{1}{ }^{1}+\theta \wedge \tau^{1}, \\
\tau^{1} & \equiv 0 \bmod \theta^{\overline{1}} \\
0 & =\theta_{1}{ }^{1}+\theta_{\overline{1}}{ }^{\overline{1}} .
\end{aligned}
$$

$\theta_{1}{ }^{1}$ and $\tau^{1}$ are called the connection form and the pseudohermitian torsion, respectively. Put $\tau^{1}=A^{1}{ }_{\overline{1}} \theta^{\overline{1}}$. The structure equation for the pseudohermitian connection is

$$
d \theta_{1}^{1}=R \theta^{1} \wedge \theta^{\overline{1}}+2 i \operatorname{Im}\left(A_{1, \overline{1}}^{\overline{1}} \theta^{1} \wedge \theta\right)
$$

where $R$ is the Tanaka-Webster curvature.

We denote components of covariant derivatives with indices preceded by a comma; thus we write $A^{\overline{1}}{ }_{1, \overline{1}} \theta^{1} \wedge \theta$. The indices $\{0,1, \overline{1}\}$ indicate derivatives with respect to $\left\{T, Z_{1}, Z_{\overline{1}}\right\}$. For derivatives of a scalar function, we omit the comma; for example, given a smooth function $\varphi$, we write $\varphi_{1}=Z_{1} \varphi$ and $\varphi_{1 \overline{1}}=Z_{\overline{1}} Z_{1} \varphi-\theta_{1}^{1}\left(Z_{\overline{1}}\right) Z_{1} \varphi$ and $\varphi_{0}=T \varphi$.

Next we recall several natural differential operators occurring in this paper. For a smooth function $\varphi$, the Cauchy-Riemann operator $\partial_{b}$ is defined locally by

$$
\partial_{b} \varphi=\varphi_{1} \theta^{1}
$$

We write $\bar{\partial}_{b}$ for the conjugate of $\partial_{b}$. A function $\varphi$ is called CR holomorphic if $\bar{\partial}_{b} \varphi=$ 0 . The divergence operator $\delta_{b}$ takes $(1,0)$-forms to functions by $\delta_{b}\left(\sigma_{1} \theta^{1}\right)=\sigma_{1}{ }^{1}$; similarly, $\bar{\delta}_{b}\left(\sigma_{\overline{1}} \theta^{\overline{1}}\right)=\sigma_{\overline{1}}{ }^{\overline{1}}$.

The Kohn Laplacian on functions is

$$
\square_{b}=2 \bar{\partial}_{b}^{*} \bar{\partial}_{b}
$$

The sublaplacian is the operator $\Delta_{b}=\operatorname{Re} \square_{b}$. The CR conformal Laplacian acts on functions $\varphi$ by $L \varphi=-\Delta_{b} \varphi+(1 / 4) R \varphi$.

The operator $P_{3}$ defined on functions $\varphi$ by $P_{3} \varphi=\left(\varphi_{\overline{1}}{ }^{\overline{1}}{ }_{1}+i A_{11} \varphi^{1}\right) \theta^{1}$ characterizes the space $\mathcal{P}$ of $\mathrm{CR}$ pluriharmonic functions as $\mathcal{P}=\operatorname{ker} P_{3}$ (see [8]). The CR Paneitz operator $P_{4}$ is defined by

$$
P_{4} \varphi=\delta_{b}(P \varphi)
$$

Define $Q$ by $Q \varphi=2 i\left(A^{11} \varphi_{1}\right)_{, 1}$. Using the commutation relation $\left[\square_{b}, \square_{b}\right]=4 i \operatorname{Im} Q$, we see that

$$
\begin{aligned}
P_{4} \varphi & =\frac{1}{4}\left(\square_{b} \bar{\square}_{b}-2 Q\right) \varphi \\
& =\frac{1}{8}\left(\left(\bar{\square}_{b} \square_{b}+\square_{b} \bar{\square}_{b}\right) \varphi+8 \operatorname{Im}\left(A^{11} \varphi_{1}\right)_{1}\right)
\end{aligned}
$$


Hence $P_{4}$ is a real and symmetric operator. Note that the leading order term of $P_{4}$ makes it a fourth order hyperbolic operator, thus it is remarkable that it still displays properties of a subelliptic operator. It follows from (1.3) that the CR pluriharmonic functions are contained in the kernel of the CR Paneitz operator. It is natural to ask whether there is anything else in the kernel of $P_{4}$. Hsiao recently showed [7] that, under the assumption that the structure $\left(M^{3}, \theta, J\right)$ is embedded, there can be at most a finite dimensional supplementary of the CR pluriharmonics in the kernel of $P_{4}$. More precisely, for any embedded structure $(M, J, \theta)$, we have

$$
\text { ker } P_{4}=\mathcal{P} \oplus W
$$

for $W$ a finite dimensional space, henceforth referred to as the supplementary space. An elementary proof of this fact is presented as Lemma 2.2 in [3]. We say that the supplementary space exists if $W \neq\{0\}$ in (1.4).

In previous work [3] on the kernel of the CR Paneitz operator, the authors showed that the supplementary space does not exist under natural conditions on an analytic family of CR manifolds.

Theorem 1.1. Let $\left(M^{3}, J^{t}, \theta\right)$ be a family of embedded $C R$ manifolds for $t \in[-1,1]$ with the following properties.

(1) $J^{t}$ is real analytic in the deformation parameter $t$.

(2) The Szegö projectors $S^{t}: F^{2,0} \rightarrow\left(\operatorname{ker} \bar{\partial}_{b}^{t} \subset F^{2,0}\right)$ vary continuously in the deformation parameter $t$ (see [5] for a definition of $F^{2,0}$ ).

(3) For the structure $J^{0}$ we have $\left\langle P_{4} \psi, \psi\right\rangle \geq 0$ for all functions $\psi$ and $\operatorname{ker} P_{4}^{0}=$ $\mathcal{P}^{0}$, the space of CR pluriharmonic functions with respect to $J^{0}$.

(4) There is a uniform constant $c>0$ such that

$$
\inf _{t \in[-1,1]} \min _{M} R^{t} \geq c>0 .
$$

(5) The CR pluriharmonic functions are stable for the family $\left(M^{3}, J^{t}, \theta\right)$. Then $P_{4}^{t} \geq 0$ and $\operatorname{ker} P_{4}^{t}=\mathcal{P}^{t}$ for all $t \in[-1,1]$.

Remark 1.2. Let $Y[J]$ denote the CR Yamabe constant of a compact CR manifold; that is,

$$
Y[J]:=\inf _{\theta}\left\{\int R \theta \wedge d \theta: \int \theta \wedge d \theta=1\right\}
$$

with the infimum taken across all contact forms on $\left(M^{3}, \xi\right)$. The assumption (1.5) can be replaced by the stronger assumption $\inf _{t} Y\left[J^{t}\right] \geq c>0$, in which case the assumptions of Theorem 1.1 are all CR invariant.

In view of [4] the hypotheses of theorem (1.2) are satisfied by the family of ellipsoids in $\mathbb{C}^{2}$.

Theorem 1.1 shows that the stability of the CR pluriharmonic functions plays a role in preventing the existence of the supplementary space. If one wishes to use deformations to exhibit examples of CR manifolds for which the supplementary space exists, one should thus look at unstable families. In the next section, we give two conditions which guarantee the existence of the supplementary space.

\section{The Supplementary Space and Instability}

Using estimates from [3], we prove two results about the supplementary space for families of embedded CR manifolds without imposing a stability assumption. 
First, using the Baire Category Theorem, we show that generically the supplementary space exists.

Theorem 2.1. Let $\left(M^{3}, J^{t}, \theta\right)$ be a family of embedded $C R$ manifolds with $t \in$ $[-1,1]$. Assume that the Szegö projector $S^{t}: F^{2,0} \rightarrow\left(\operatorname{ker} \bar{\partial}_{b}^{t} \subset F^{2,0}\right)$ varies real analytically in the deformation variable $t$ (see [5] for a definition of $F^{2,0}$ ). Then

(1) $n_{0}:=\sup _{t \in[-1,1]} \operatorname{dim} W^{t}<\infty$.

(2) The set

$$
F:=\left\{t \in[-1,1]: \operatorname{dim} W^{t} \leq n_{0}-1\right\}
$$

is a closed set with no accumulation points. In particular, $F$ has no interior points and hence $F$ is of the first category.

(3) The set

$$
E:=\left\{t \in[-1,1]: \operatorname{dim} W^{t}=n_{0}\right\}
$$

has nonempty interior.

Remark 2.2. The theorem above states that for generic values $t$ of the deformation parameter, $\operatorname{dim} W^{t}=n_{0}$. Since $n_{0}>0$ if there exists a $t_{0} \in[-1,1]$ with $W^{t_{0}} \neq\{0\}$, the supplementary space exists for a generic value of $t$ if it exists for some $t_{0}$. Moreover, if $n_{0}>0$, then $\operatorname{dim} W^{t}=0$ for a thin set $F \subset[-1,1]$ of the first category.

Proof. To prove the theorem we apply [1, Lemma 2.28]. The statement of $[1$, Lemma 2.28] is for deformation parameters that are complex analytic, but we here apply it to the finite rank operators $A^{t}$ constructed in [3, Lemma 2.9] which depend the real variable $t$. The assumption that $J^{t}$ and $S^{t}$ depend real-analytically on $t$ yields that the function $h(t)=\left\langle A^{t} \phi, \psi\right\rangle$ from the proof of [3, Lemma 2.9] is real analytic in $t$. Thus the zeros of $h(t)$ are isolated and we conclude exactly as in $[1$, Lemma 2.28] that

$$
\sup _{t \in[-1,1]} \operatorname{rank} A^{t}=\sup _{t \in[-1,1]} \operatorname{dim} W^{t}<\infty,
$$

where we use [3, Lemma 2.8] to establish the first equality. This proves the first claim.

Next, [3, Corollary 2.11] implies that $\operatorname{dim} W^{t}$ is lower semi-continuous, and thus the set $F$ is closed. By the conclusion of [1, Lemma 2.28], the set

$$
\left\{t \in[-1,1]: \operatorname{rank} A^{t}<n_{0}\right\}=\left\{t \in[-1,1]: \operatorname{rank} A^{t} \leq n_{0}-1\right\}
$$

has no accumulation points. This and [3, Lemma 2.8] yield the second claim.

Finally, note that

$$
\left\{t \in[-1,1]: \operatorname{dim} W^{t}>n_{0}-1\right\}=\left\{t \in[-1,1]: \operatorname{rank} A^{t}>n_{0}-1\right\}
$$

cannot be empty; if it were, this would contradict the definition of $n_{0}$. Since $\operatorname{dim} W^{t}$ is lower semi-continuous, the sets (2.1) are open and nonempty, yielding the last claim.

Second, we show that, under an assumption on the rate of vanishing of the first eigenvalue of the CR Paneitz operator for a family of CR structures, the loss of stability of the CR pluriharmonic functions implies the existence of the supplementary space. To make this precise, we list our assumptions. 
Let $\left(M^{3}, J^{t}, \theta\right)=: M^{t}$ be a family of embedded CR manifolds for which $J^{t}$ is $C^{6}$ in the deformation parameter $t$ for some interval $\left|t-t_{0}\right|<\mu$ with $\mu>0$. Suppose that there is a constant $c>0$ independent of $t$, such that:

(1) For any $t \neq t_{0}$ and any $f \perp \operatorname{ker} P_{4}^{t}$, it holds that

$$
\left|t-t_{0}\right| \eta\left(\left|t-t_{0}\right|\right)\|f\|_{2} \leq\left\|P_{4}^{t} f\right\|_{2}
$$

where $\eta(s) \rightarrow \infty$ as $s$ tends to zero.

(2) For any $f \perp \operatorname{ker} P_{4}^{t_{0}}$, it holds that

$$
c\|f\|_{2} \leq\left\|P_{4}^{t_{0}} f\right\|_{2} .
$$

Note that the inequality $(2.2 \mathrm{~b})$ was observed by Cao and Chang [2] for a fixed embedded CR manifold. Together, the assumptions (2.2a) and (2.2b) imply that the lowest nonzero absolute value of the eigenvalues of the CR Paneitz operator $P_{4}^{t}$ jumps up as $t \rightarrow t_{0}$.

Next, we assume there is a family of diffeomorphisms $\Phi^{t}: M^{t} \rightarrow M^{0}:=M^{t_{0}}$ which is $C^{6}$ in the deformation parameter $t$ and is such that $\Phi^{0}$ is the identity map.

Lastly, we assume that the CR pluriharmonic functions are unstable at $t_{0}$. More precisely, we assume that there is a CR pluriharmonic function $f_{0} \in C^{5}$ for the structure $M^{0}$ and constants $\varepsilon>0$ and $0<\delta<\mu$ such that for any $t$ with $\left|t-t_{0}\right|<\delta$ and any CR pluriharmonic function $\psi \in \mathcal{P}^{t}$, it holds that

$$
\left\|f_{0}-\psi\right\|_{2} \geq \varepsilon \text {. }
$$

Theorem 2.3. Assume $\left(M^{3}, J^{t}, \theta\right)$ is as described above. Then for all $t \neq t_{0}$ with $\left|t-t_{0}\right|<\delta$ and $\delta$ sufficiently small, the supplementary space $W^{t}$ exists.

Proof. Set $u_{t}=f_{0} \circ \Phi^{t}$. By assumption, $u_{t} \in C^{5}$. We claim that $u_{t}$ is not perpendicular to $\operatorname{ker} P_{4}^{t}$. If instead $u_{t} \perp \operatorname{ker} P_{4}^{t}$, then (2.2a) gives

$$
\left|t-t_{0}\right| \eta\left(\left|t-t_{0}\right|\right)\left\|u_{t}\right\|_{2} \leq\left\|P_{4}^{t} u_{t}\right\|_{2} .
$$

On the other hand, using the fact that $f_{0} \in \mathcal{P}^{t_{0}}$, we have that

$$
\left\|P_{4}^{t} u_{t}\right\|_{2} \leq\left\|\left(P_{4}^{t}-P_{4}^{t_{0}}\right) u_{t}\right\|_{2}+\left\|P_{4}^{t_{0}}\left(u_{t}-f_{0}\right)\right\|_{2} .
$$

Applying (2.4) and the definitions of $\Phi^{t}$ and $u_{t}$ thus yields

$$
\left|t-t_{0}\right| \eta\left(\left|t-t_{0}\right|\right)\left\|u_{t}\right\|_{2} \leq c\left|t-t_{0}\right| .
$$

Recalling that $\eta\left(\left|t-t_{0}\right|\right) \rightarrow \infty$ as $t \rightarrow t_{0}$, we have that $\left\|u_{0}\right\|_{2}=\left\|f_{0}\right\|_{2}=0$, a contradiction.

Now assume that the projection of $u_{t}$ onto ker $P_{4}^{t}$ is a CR pluriharmonic function $\psi_{t} \in \mathcal{P}^{t}$. We may write

$$
u_{t}=v_{t}+\psi_{t}
$$

with $v_{t} \perp \operatorname{ker} P_{4}^{t}$. Since $\psi_{t}$ is CR pluriharmonic,

$$
P_{4}^{t} u_{t}=P_{4}^{t} v_{t} .
$$

On the other hand, by using the fact that $f_{0} \in \mathcal{P}^{t_{0}}$ as above, we find that

$$
\left\|P_{4}^{t} u_{t}\right\|_{2} \leq c\left|t-t_{0}\right| \text {. }
$$

Since $v_{t} \perp \operatorname{ker} P_{4}^{t}$ we may apply (2.2) together with (2.6) and (2.7) to find that

$$
\left|t-t_{0}\right| \eta\left(\left|t-t_{0}\right|\right)\left\|v_{t}\right\|_{2} \leq\left\|P_{4}^{t} v_{t}\right\|_{2} \leq c\left|t-t_{0}\right| .
$$


In particular,

$$
\left\|v_{t}\right\|_{2}<\varepsilon
$$

for $\left|t-t_{0}\right|<\delta$. Now,

$$
f_{0}-\psi_{t}=f_{0}-u_{t}+v_{t} .
$$

Hence

$$
\left\|f_{0}-\psi_{t}\right\|_{2} \leq\left\|f_{0}-u_{t}\right\|_{2}+\left\|v_{t}\right\|_{2} .
$$

By (2.8) and the construction of $u_{t}$, we conclude that $\left\|f_{0}-\psi_{t}\right\|_{2}=o(1)$ for $t \rightarrow t_{0}$. This contradicts the stability assumption (2.3). Hence the projection of $u_{t}$ onto ker $P_{4}^{t}$ cannot be a CR pluriharmonic function, whence $W^{t} \neq\{0\}$.

\section{REFERENCES}

[1] D. M. Burns and C. L. Epstein. Embeddability for three-dimensional CR-manifolds. J. Amer. Math. Soc., 3(4):809-841, 1990.

[2] J. Cao and S.-C. Chang. Pseudo-Einstein and $Q$-flat metrics with eigenvalue estimates on CR-hypersurfaces. Indiana Univ. Math. J., 56(6):2839-2857, 2007.

[3] J. S. Case, S. Chanillo, and P. Yang. The CR Paneitz operator and the stability of CR pluriharmonic functions. arXiv:1502.01994, preprint.

[4] S. Chanillo, H.-L. Chiu, and P. Yang. Embedded three-dimensional CR manifolds and the nonnegativity of Paneitz operators. In Geometric analysis, mathematical relativity, and nonlinear partial differential equations, volume 599 of Contemp. Math., pages 65-82. Amer. Math. Soc., Providence, RI, 2013.

[5] P. M. Garfield and J. M. Lee. The Rumin complex on CR manifolds. Sūrikaisekikenkyūsho Kōkyūroku, (1037):29-36, 1998. CR geometry and isolated singularities (Japanese) (Kyoto, 1996).

[6] C. R. Graham and J. M. Lee. Smooth solutions of degenerate Laplacians on strictly pseudoconvex domains. Duke Math. J., 57(3):697-720, 1988.

[7] C.-Y. Hsiao. On CR Paneitz operators and CR pluriharmonic functions. arXiv:1405.0158, preprint.

[8] J. M. Lee. Pseudo-Einstein structures on CR manifolds. Amer. J. Math., 110(1):157-178, 1988.

Department of Mathematics, Princeton University, Princeton, NJ 08540

E-mail address: jscase@math.princeton.edu

Department of Mathematics, Rutgers University, 110 Frelinghuysen Rd., Piscataway, NJ 08854

E-mail address: chanillo@math.rutgers.edu

Department of Mathematics, Princeton University, Princeton, NJ 08540

E-mail address: yang@math.princeton.edu 